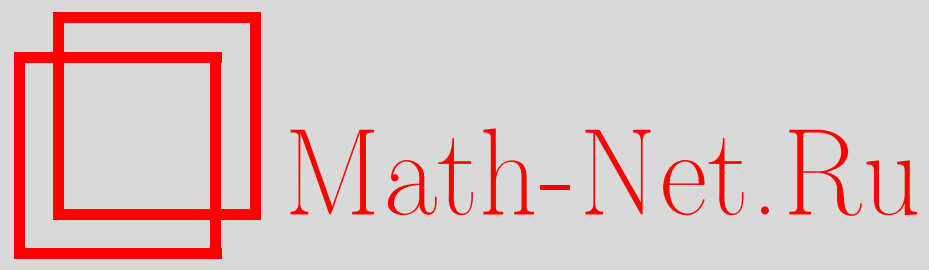

С. И. Яковлев, О сингулярном спектре операторов модели Фридрихса в окрестности особой точки, Функи. анализ и его прил., 1998, том 32, выпуск $3,91-94$

DOI: https://doi.org/10.4213/faa434

Использование Общероссийского математического портала MathNet.Ru подразумевает, что вы прочитали и согласны с пользовательским соглашением http://www.mathnet.ru/rus/agreement

Параметры загрузки:

IP: 52.23 .180 .231

26 апреля 2023 г., 06:24:14

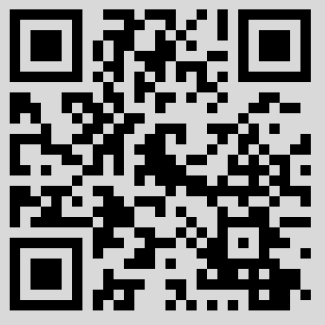




\title{
О сингулярном спектре операторов модели Фридрихса в окрестности особой точки
}

\author{
(c) 1998. С. И. Яковлев
}

Рассмотрим самосопряженные операторы $a_{m}$ и $s_{m}, m=1,2, \ldots$, действующие в пространстве $L_{2}(\mathbb{R})$,

$$
\begin{aligned}
a_{m} & =|t|^{m} \cdot+(\cdot, \varphi) \varphi, \\
s_{m} & =\operatorname{sign} t \cdot|t|^{m} \cdot+(\cdot, \varphi) \varphi,
\end{aligned}
$$

где $\varphi \in L_{2}(\mathbb{R})$, а $t$ - независимая переменная, так что, например,

$$
\left(a_{m} u\right)(t)=|t|^{m} u(t)+\varphi(t) \int_{\mathbb{R}} u(x) \overline{\varphi(x)} d x, \quad u \in L_{2}(\mathbb{R}) .
$$

Предполагается, что функция $\varphi$ удовлетворяет условию гладкости

$$
|\varphi(t+h)-\varphi(t)| \leqslant \omega(|h|), \quad|h| \leqslant 1,
$$

где функция $\omega(t)$ (модуль непрерывности функции $\varphi$ ) монотонна и удовлетворяет условию Дини:

$$
\omega(t) \downarrow 0 \quad \text { при } t \downarrow 0 \quad \text { и } \quad \int_{0}^{1} \frac{\omega(t)}{t} d t<\infty .
$$

Нас интересует поведение сингулярного спектра операторов $a_{m}$ и $s_{m}$. Заметим, что под сингулярным спектром мы понимаем объединение сингулярного непрерывного и точечного спектров. Структура спектра $\sigma_{\operatorname{sing}}\left(s_{1}\right)$ (сингулярного спектра оператора $s_{1}$ ) достаточно хорошо изучена [1-8]. Но, сделав простую замену переменных $|t|^{m}=x$ или $\operatorname{sign} t \cdot|t|^{m}=x$, можно показать, что вне любой окрестности точки нуль структура спектров $\sigma_{\text {sing }}\left(a_{m}\right)$ и $\sigma_{\text {sing }}\left(s_{m}\right)$ такая же, как и у оператора $s_{1}$. Это объясняется тем, что указанные замены переменных вне любой окрестности точки нуль гладкие. При этом если условия (3), (4), а так же некоторые другие ограничения на функцию $\varphi$ выполняются только на некотором интервале $(c, d)$ вешественной оси, то основные результаты работ [1-8], относящиеся к структуре спектра $\sigma_{\text {sing }}\left(s_{1}\right)$, останутся справедливыми на любом замкнутом подынтервале $\Delta \subset(c, d)$. В то же время, как показано в $[9,10]$, для оператора $a_{2}$ в окрестности точки нуль поведение сингулярного спектра приобретает совершенно иной характер. Здесь указанную замену переменных тоже можно применить, но, поскольку $\left.\left(|t|^{m}\right)^{\prime}\right|_{0}=\left.\left(\operatorname{sign} t \cdot|t|^{m}\right)^{\prime}\right|_{0}=0$, она не является гладкой (диффеоморфизмом) в окрестности точки нуль. В этом смысле точка нуль является особой точкой операторов $a_{m}$ и $s_{m}, m>1$. Естественно, что все наше внимание будет обращено на сингулярный спектр именно в окрестности этой особой точки. Отметим также, что для операторов $a_{m}$ точка нуль является, кроме того, крайней точкой непрерывного спектра, совпадающего с 
интервалом $[0 ;+\infty)$. Для операторов $s_{m}$ непрерывный спектр заполняет всю вещественную ось $\mathbb{R}$.

Начнем с построения функции $\varphi(t)$, такой, что операторы $a_{m}$ и $s_{m}$ имеют нетривиальный сингулярный спектр около особой точки нуль. Заметим, что настоящий модуль непрерывности $\widetilde{\omega}(h):=\sup _{t}|\varphi(t+h)-\varphi(t)|$ всегда удовлетворяет дополнительному ограничению полуаддитивности $\widetilde{\omega}\left(t_{1}+t_{2}\right) \leqslant$ $\widetilde{\omega}\left(t_{1}\right)+\widetilde{\omega}\left(t_{2}\right)$.

ТЕОРЕмА 1. Пусть функиия $\omega(t)$ удовлетворяет условию (4), а также естественному ограничению полуаддитивности $\omega\left(t_{1}+t_{2}\right) \leqslant \omega\left(t_{1}\right)+\omega\left(t_{2}\right)$; тогда, если $\overline{\lim }_{t \rightarrow 0} \omega^{2}(t) / t^{m-1}=+\infty$, можно построить финитную функцию $\varphi$, удовлетворяюшую условию $|\varphi(t+h)-\varphi(t)| \leqslant \omega(|h|)$ u такую, что операторь $a_{m}$ u $s_{m}$ имеют сходяшуюся $\kappa$ точке нуль последовательность собственных значений, расположенных на непрерывном спектре.

СлЕДСТВИЕ 1. Нетрудно показать, что если $\omega(t)$ - неотрииательная полуаддитивная функиия $u \omega(t) \downarrow 0$ при $t \downarrow 0$, то для любого $а>0$ сушествует константа $C>0$, такая, что $C t \leqslant \omega(t), t \in[0, a]$. Поэтому, как следует из теоремь 1, для любого $m \geqslant 4$ и всякой полуаддитивной функиии $\omega(t)$, удовлетворяющей условию (4), можно построить финитную функиию $\varphi$, удовлетворяюшую условию гладкости (3) и такую, что операторьл $a_{m} \quad u \quad s_{m}$ имеют последовательность положительных собственных значений, сходящуюся к точке нуль.

Чтобы выяснить значение приведенных выше утверждений, рассмотрим в пространстве $L_{2}(\mathbb{R})$ самосопряженные операторы $A_{m}$ и $S_{m}$,

$$
\begin{aligned}
& A_{m}=|t|^{m} \cdot+V, \\
& S_{m}=\operatorname{sign} t \cdot|t|^{m} \cdot+V,
\end{aligned}
$$

где $V$ (возмущение) 一 интегральный оператор с эрмитовым ядром $v(t, x)$, удовлетворяющим следующему условию гладкости:

$$
v(t+h, t+h)+v(t, t)-v(t+h, t)-v(t, t+h) \leqslant \omega^{2}(|h|), \quad|h| \leqslant 1,
$$

с функцией $\omega(t)$ типа (4). Мы также предполагаем, что оператор $V$ неотрицателен и принадлежит классу ядерных операторов $\mathfrak{S}_{1}$ :

$$
V \geqslant 0, \quad V \in \mathfrak{S}_{1} .
$$

При этом (7) можно рассматривать как условие гладкости на ядро $v_{1 / 2}(t, x)$ оператора $\sqrt{V}$, ибо левая часть выражения (7) тождественно переписывается [3] в следующем виде: $\int_{\mathbb{R}}\left|v_{1 / 2}(t+h, y)-v_{1 / 2}(t, y)\right|^{2} d y$. Очевидно, что для $V=(\cdot, \varphi) \varphi$ операторы $A_{m}$ и $S_{m}$ совпадают соответственно с $a_{m}$ и $s_{m}$, определяемыми формулами (1) и $(2)$.

В работе [8] (см. также [6]) показано, что для оператора $S_{1}$ имеется точное условие конечности сингулярного спектра. А именно, если $\omega(t)=O(\sqrt{t})$ при $t \rightarrow 0$, то при условиях $(7),(8)$ спектр $\sigma_{\operatorname{sing}}\left(S_{1}\right)$ тривиален, т. е. состоит не более, чем из конечного числа собственных значений конечной кратности. Если же $\varlimsup_{\lim _{t \rightarrow 0} \omega}(t) / \sqrt{t}=+\infty$, то строятся примеры, показывающие, что 
уже в случае возмущения $V$ ранга 1 у оператора $S_{1}$ могут появиться точки накопления собственных значений. Как мы видим, согласно следствию 1, для операторов $A_{m}$ и $S_{m}$ при $m>3$ отсутствует условие конечности сингулярного спектра в классе возмущений, описываемом условиями $(7),(8)$.

В работе [10] (см. также [9]) было показано, что если $\omega(t)=O(\sqrt{t})$ при $t \rightarrow 0$ и $\operatorname{rank} V<\infty$, то особая точка нуль не является точкой сгущения сингулярного спектра оператора $A_{2}=t^{2} \cdot+V$. Следовательно, согласно теореме 1 , при $m=2$ это условие $\omega(t)=O(\sqrt{t}), t \rightarrow 0$, является точным в случае, когда $\operatorname{rank} V<\infty$. (При $\operatorname{rank} V=\infty$ значение константы $C$ в условии $\omega(t)=C t^{1 / 2}, t \rightarrow 0$, определяет, конечен $\sigma_{\operatorname{sing}}\left(A_{2}\right)$ или же он может иметь сложную структуру $[9,10]$.$) Заметим, что хотя для оператора S_{1}=t \cdot+V$ точным условием конечности спектра $\sigma_{\operatorname{sing}}$ также является оценка $\omega(t)=$ $O(\sqrt{t}), t \rightarrow 0$ (независимо от ранга оператора возмущения) [6, 8], контрпример из работы [8], подтверждающий точность данного условия, принципиально нельзя приспособить к случаю особой точки нуль оператора $A_{2}$.

Как показывает теорема 1 при $m=3$, невыполнение условия $\omega(t)=O(t)$, $t \rightarrow 0$, ведет к возможности появления у операторов $A_{3}$ и $S_{3}$ в окрестности особой точки нуль нетривиального сингулярного спектра. В приводимой ниже теореме 2 показывается, что для возмущения конечного ранга, $\operatorname{rank} V<\infty$, условие $\omega(t)=O(t), t \rightarrow 0$, является достаточным для отсутствия спектра $\sigma_{\operatorname{sing}}\left(A_{3}\right)$ около точки нуль. Согласно теореме 1 , это условие является точным.

ТЕорема 2. Пусть оператор $V$ удовлетворяет условиям (7), (8) с функиией $\omega(t)$, такой, что $\omega(t)=O(t)$ при $t \rightarrow 0, u \operatorname{rank} V<\infty ;$ тогда в некоторой окрестности точки нуль (на интервале $[0 ;+\infty))$ спектр оператора $A_{3}=|t|^{3} \cdot+V$ чисто абсолютно непрерывный.

В то же время в случае $\operatorname{rank} V=\infty$ ответ на вопрос, конечен сингулярный спектр или же он может иметь сложную структуру, зависит от малости константы $C$ в условии $\omega(t)=C t, t \rightarrow 0$.

ТЕОРЕмА 3. Пусть возмущение $V$ удовлетворяет условиям (7), (8) $c$ функией $\omega(t)$, такой, что $\omega(t)=C t$ при $t \rightarrow 0 ;$ тогда, если константа $C$ меньше 1 , спектр оператора $A_{3}$ в некоторой окрестности точки нуль чисто абсолютно непрерьвный.

Следовательно, при выполнении условий теоремы 2 или 3 спектр $\sigma_{\operatorname{sing}}\left(A_{3}\right)$ будет тривиален. Точность теоремы 3 подтверждает теорема 4.

ТеоремА 4. Для значения константь $C \geqslant 3$ можно построить операmор $V \geqslant 0, V \in \mathfrak{S}_{1} u \operatorname{rank} V=\infty$, удовлетворяюший условию $(7)$ с модулем непрерьвности $\omega(t), \omega(t)=C t$ при $t \rightarrow 0$, такой, что операторь $A_{3} u S_{3}$ имеют последовательность положительных собственньх значении, сходяшуюся к точке нуль.

Таким образом, для оператора $A_{3}$, как и для оператора $A_{2}[9,10]$, точное условие конечности спектра $\sigma_{\text {sing }}$ имеет разный вид для возмущений конечного и бесконечного рангов. Примеры операторов возмущения $V$ бесконечного ранга, приводящие к нетривиальному сингулярному спектру около особой точки нуль, содержатся в теореме 5, которая также подтверждает 
результат следствия 1 об отсутствии условия конечности $\sigma_{\operatorname{sing}}$ у операторов $A_{m}$ и $S_{m}$ при $m>3$ в классе возмущений, удовлетворяющих требованиям (7), (8).

ТЕОРема 5. Для любой константы $C>0$ и любого натурального $m \geqslant 4$ можно построить оператор возмущения $V \geqslant 0, V \in \mathfrak{S}_{1} u \operatorname{rank} V=\infty$, удовлетворяющий условию гладкости (7) с модулем непрерывности $\omega(t)$, $\omega(t)=C t$ при $t \rightarrow 0$, такой, что операторы $A_{m} u S_{m}$ имеют последовательность собственных значений, сходящуюся $\kappa$ точке нуль.

Следующий простой результат завершает изучение вопроса о границе конечности сингулярного спектра операторов $A_{m}, m=1,2, \ldots$.

Теорема 6. Пусть оператор $V$ удовлетворяет условиям (7), (8); тогда при любом модуле непрерьвности $\omega(t)$, удовлетворяюшим условию (4), спектр оператора $A_{1}=|t| \cdot+V$ в некоторой окрестности точки нуль чисто абсолютно непрерьвньй.

Для операторов $S_{2}$ и $S_{3}$, согласно теореме 1 , конечности спектра $\sigma_{\operatorname{sing}}$ можно ожидать только при выполнении условий $\omega(t)=O\left(t^{1 / 2}\right)$ и $\omega(t)=O(t)$ соответственно. Мы полагаем, что на самом деле ситуация с операторами $S_{2}$ и $S_{3}$ такая же, как и с операторами $S_{m}, m>3$, т. е. условие конечности сингулярного спектра для этих двух операторов также отсутствует.

В заключение автор благодарит С. Н. Набоко за внимание к работе.

\section{ЛиТеРАТУРА}

1. Фаддеев Л. Д. Труды МИАН, 73, 292-313 (1964). 2. Павлов Б. С., Пеmpac C. В. Функц. анализ и его прил., 4, вып. 2, 54-61 (1970). 3. Naboko S. N. Ark. Mat., 25, №. 1, 115-140 (1987). 4. Микитюк Я. В. ДАН СССР, 303, № 1, 33-36 (1988). 5. Яковлев С. И. Вестник ЛГУ, сер. 1, № 1, 116-117 (1990). 6. Набоко С. Н., Яковлев С. И. Функц. анализ и его прил., 24, вып. 4, 88-89 (1990). 7. Яковлев С. И. Деп. в ВИНИТИ, № 2050-В от 17.05.91. 8. Дьнькин Е. М., Набоко C. Н., Яковлев С. И. Алгебра и анализ, 3, вып. 2. 77-90 (1991). 9. Yakovlev S. I. Preprint IPRT No. 48-94, December 1994. 10. Яковлев С. И. Функц. анализ и его прил., 30, вып. 1, 92-95 (1996).

Санкт-Петербургская академия аэрокосмического приборостроения e-mail: yakovlev@amath.usr.saai.ru
Поступило в редакцию 8 апреля 1997 г. 